\title{
Determination of Chemoattractant factor (Interleukin -8) and Gamma interferon (IFN- $\square$ ) among Lymphoma patients (Hodgkin's and Non Hodgkin).
}

\author{
Raheem Tuama O. AL-Mammori ${ }^{1}$, Dr . Azhar Emran AL-Thahab . ${ }^{2}$ Dr . Alaa \\ Sadeq Al-Awad. ${ }^{3}$ \\ ${ }^{1}$ (Msc. Clinical immunology. Biology Department/Babylon university/ Science College/ Iraq. ) \\ ${ }^{2}$ (Assist. prof/Immunology. Biology Department /Babylon university/Science College/Iraq . ) \\ ${ }^{3}$ (Assist. prof/ Consultant physician. Babylon University/ Medicine college/ Iraq. )
}

\begin{abstract}
Fifty (50) patients clinically diagnosed as lymphoma patients, 17 (34\%) Hodgkin's and, 33 (66\%) patients Non Hodgkin attending Tumor Center in Merjan medical city, and (30) matched age person, as a control group enrolled in this study in the period between Junuary and Augest 2012. Specific diagnosis is clinically done by consultant physician, histopathologist and confirmed by specific marker or Cluster of Differentiation (CD) markers (CD20 and CD30), IL-8, and IFN- $\gamma$, estimated for all patients and control. The result shows that a highly significant $(P<0.01)$ increased in IFN- $\gamma$ in both groups $H L$, and $N H L$, compared with control . IL-8 shows highly significant decreased $(P<0.01)$ in result of NHL, compared with control, as well as no significant decreased of $H L$, in comparison with control. There is significant increased $(P<0.05)$ in IL-8 level of HL patients compared with NHL. The Immunohistochemical result shows that highly association between CD2O with NHL, CD30 with HL. And it used specifically to confirm histopathological diagnosis of lymphomas. From present study can concluding the facts that, Activation of B and T cells in lymphoma patients lead to increased anti proliferating activity and immunomodulary of IFN- $\gamma$, and in less extent IL-8 as chemotactic factor. Cytokines level important to monitored the severity of damage and whether the patients with good or bad prognosis. So that recommended to study other different types of cytokines for Th1, Th2, and NK cell activators to predict of ability to producing pro-inflammatory and anti-inflammatory mediators as well as ability to treatment of such diseases.
\end{abstract}

Keywords:Chemotactic Factor. Hodgkin's lymphoma, Interleukin-8, Interferon- $\gamma$, Non Hodgkin's lymphoma.

\section{Introduction.}

Lymphoma is the most common cancer of childhood in many parts of the world. The infection rate may be even higher than other malignancies. The exact mechanism cause Hodgkin's lymphoma is not well understood, but it is becoming very evident that EBV has an important role to play in the development of this lymphoma. Other type is Non Hodgkin's lymphoma also may associated with lymphoadenpathy and certain types of autoimmunity [1].

\subsection{Cytokines And Lymphoma.}

Interleukin -12 (IL-12) is a heterodimeric cytokines that induce interferon -gamma (INF- $\gamma$ ) production by natural killer cells and T-lymphocytes, also activate human B-cell, through the IL-12 receptor (IL-12 R) complex.[2]. Since Interleukin-8 functions both as a leukocyte and lymphocyte chemoattractant, by biopsy from cutaneous T-cell lymphoma (CTCL). These results were corroborated by strong hybridization signals using IL-8 probes in only the lesional CTCL skin. Lesional CTCL epidermis contains elevated levels of IL-8 mRNA and protein suggesting a role for this cytokine in the pathogenesis of the disease.[3].

\subsection{Cluster Of Diffrentistion ( Cd Marker) In Lymphoma Patients.}

The hallmark cells (and variants) show immunopositivity for CD30. True positivity requires localization of signal to the cell membrane and/or para nuclear region (cytoplasmic positivity is considered nonspecific and non-informative). Another useful marker which helps to differentiate this lesion from Hodgkin lymphoma is clusterin. The neoplastic cells have a golgi staining pattern (hence para nuclear staining), which is characteristic of this lymphoma. The cells are also typically positive for a subset of markers of T-cell lineage. However, as with other T-cell lymphomas, they are usually negative for the pan T-cell marker CD3. Occasional examples are of null (neither T nor B) cell type.[4,5]. The CD20 antigen, glycoprotein found on all B cells in peripheral blood, lymph node, spleen, tonsil, and bone marrow, normal cells do not express the CD20 antigen. 
This antibody may be used in the study of normal and neoplastic cells of B cell origin, in the study of B cell development, and activation. [6].

The product of fusion gene may be identified by immunohistochemstry using antiserum to ALK protein. Probes are available to identify the translocation by fluorescent in situ hybridization. The nucleophosmin component associated with the commonest translocation results in nuclear positivity as well as cytoplasmic positivity. Positivity with the other translocations may be confined to the cytoplasm.[7].

\section{Patients And Methods.}

Fifty (50) patients clinically diagnosed as lymphoma patients, 17 (34\%) Hodgkin's and 33 (66\%) patients, Non Hodgkin, attending tumor center in merjan medical city and (30) matched age person, as a control group enrolled in this study in the period between Junuary and Augest 2012. Specific diagnosis is clinically done by consultant physician, histopathologist and confirmed by specific marker or Cluster of Differentiation (CD) markers (CD20 and CD30) done by using immunohistochemical technique (IHC) according to manual principle and method of Dakocytomation company [8]. IL-8, IFN- $\gamma$, also done by ELISA technique for all patients and control groups, by using manual principle and methods of BioSource Company.[9].

\section{Result And Discussion.}

Cytokines level important to monitored the severity of damage and whether the patients with good or bad prognosis, so that recommended to study different types of cytokines for Th1,Th2 and NK cell activators. Increased level of interferon is constituent with disease requirement because the interferon used in regulation and maintenance of immunological response, as well as used as therapeutic agent for different diseases from which, is lymphomas. The result of present study shows that a highly significant $(\mathrm{P}<0.01)$ increased in mean result of IFN- $\gamma$ in both groups HL, $682 \mathrm{pg} / \mathrm{ml}$ and NHL, $760 \mathrm{pg} / \mathrm{ml}$ in compareson with control group, 404.3 $\mathrm{pg} / \mathrm{ml}$. as mentioned in "Fig. (1)" . IFN- $\gamma$ inhibits the activity of Th2 cells, thereby inhibiting antibody synthesis. It is also the most potent activator of macrophages. It stimulates MHC class II expression on macrophage lineage cells and endothelial cells. IFN- $\gamma$ used as antiviral, antiprolefreative and immunomodulary effects.[10]. Significantly increased CD4+, IFN-gamma producing (Th1) cell percentage were found in untreated lymphoma cases. CD8+ IL-4 and IFN-gamma producing (Tc0) T cell frequency is significantly higher in untreated lymphoma patients compared with normal controls. Patients in long-term remission have lower frequency of CD4+, IL4 producing (Th2) cell, and increased CD4+ IFN-gamma producing (Th1) cell frequency, compared with healthy normal controls. These may contribute to strong polarization toward Th1 type response, needed for lymphoma clearance and remission.[11].

IL-8 small ,inducible, secreted cytokines or chemoattractant or super family of low molecular weight proteins. Play an important role as pathogenic mediators in several pathogenesis, inflammatory and autoimmune diseases. IL-8 shows highly significant decreased $(\mathrm{P}<0.01)$ in mean concentration in NHL, $143.48 \mathrm{pg} / \mathrm{ml}$ as compared with control samples, as well as no significant decreased in mean concentration of HL, $265.1 \mathrm{pg} / \mathrm{ml}$ in comparison with control .There is significant increased $(\mathrm{P}<0.05)$ in IL-8 level of $\mathrm{HL}$ patients compared with NHL. As shown in "Fig. (2)". Except TNF alpha, significantly higher pretreatment levels of interleukin-2(IL-2), soluble interleukin-2 receptor (sIL-2R), interleukin-6 (IL-6) and interleukin-8 (IL-8) were observed in most of Malignant lymphoma (ML) patients at diagnosis or relapse as compared with controls. Cytokine levels declined in responding patients after therapy and there were no differences between, the cytokine levels remained elevated in non responding patients. The elevated IL-8 level correlated with the clinical stage and presenting B symptoms of ML patients and had no correlation with other clinical-hematological parameters. The changes of cytokines may be served as a means to observe the condition of ML patients and supervise their response to treatment.[12]. Cytokines play important roles in the pathogenesis of lymphomas. Elevated levels of IL-6, IL-8 and IL-10 correlated with more adverse disease features. Consequently, patients with elevated IL-6, IL-8 and IL-10 levels prior to treatment had a lower response to therapy. Furthermore, those with elevated IL-6 and IL-10 levels had poor median, 3-year and 5-year survival, while elevated serum IL-8 level did not correlate with overall survival. Worse survival was also confirmed in patients with combined elevated pretreatment serum levels of IL-6, IL-8 and IL-10. Serum levels of IL-6, IL-8 and IL-10 before treatment of patients with newly diagnosed Diffused large B- cell lymphoma (DLBCL) may give some insight into the possible prognosis and thus facilitate the decisions regarding therapeutic approaches for individual patients. [13].

Lymphoid immune status contribute with the higher level of such cytokines as a monitor for progressive pathway of diseases. The result of present study shows that, there is a highly association between CD20 with NHL, $29: 33$ ( 87.8\%) , CD30 with HL, 12:17 ( 70.8\%) as shown in "Fig. (3)". CD30, is glycoprotein, expressed in only a small population of normal lymphoid tissue. By contrast, it is expressed in approximately $50 \%$ of all malignant lymphomas including all cases of Hodgkin's disease and a vast majority of anaplastic large cell lymphomas. Antigen can be detected in sera from lymphoma patients, but not in sera from 
normal individuals with systemic infection. The CD20 antigen, glycoprotein found on all B cells in peripheral blood, lymph node, spleen, tonsil, and bone marrow, normal cells do not express the CD20 antigen. This antibody may be used in the study of normal and neoplastic cells of B cell origin, in the study of B cell development, and activation.[6]

CD30 (Ki-1 antigen), a $120 \mathrm{kD}$ single chain glycoprotein, is expressed in only a small population of normal lymphoid tissue. By contrast, it is expressed in approximately $50 \%$ of all malignant lymphomas including all cases of Hodgkin's disease and positive anaplastic large cell lymphomas. Ki-1 antigen can be detected in sera from lymphoma patients, but not in sera from normal individuals with systemic infection. The antibody reacts with a formalin-resistant intracytoplasmic epitope found in the majority of B cells which is now considered to be the CD20 antigen, a pan-B cell marker. The antibody primarily recognizes a polypeptide $\mathrm{B}$ cell component and also a minor cellular antigen. The staining pattern is consistent with pan-B reactivity, producing staining for B cells in lymphoid and peripheral blood tissue. This antibody may be used in the study of normal and neoplastic cells of B origin, in the study of B cell development and activation [14].

\section{V. Figures}

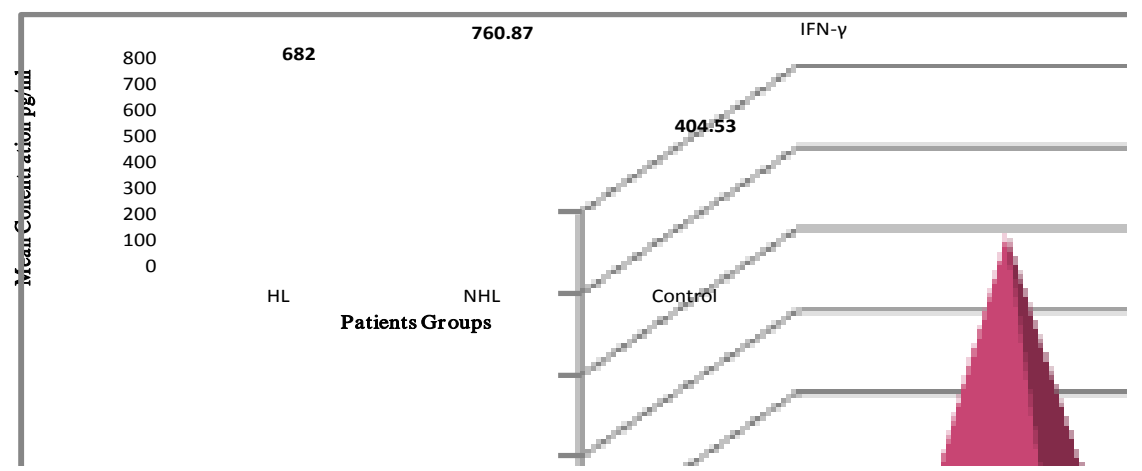

Figure (1) Concentration of IFN- $\gamma$ of lymphoma patients ( $\mathrm{H}$ and $\mathrm{NH}$ ) with control.

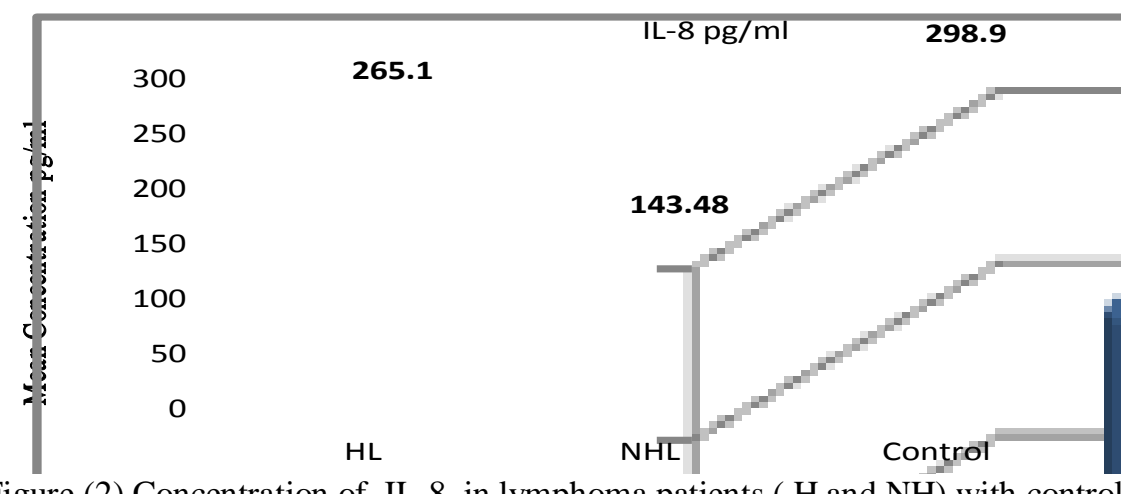

Figure (2) Concentration of IL-8 in lymphoma patients ( $\mathrm{H}$ and $\mathrm{NH}$ ) with control.

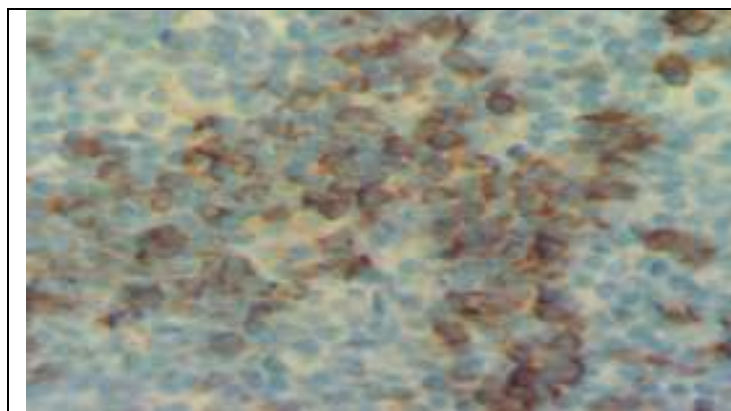

CD 20 , positive marker in $19: 33$ ( $87.8 \%)$ of NHL , appear as brownish color surrounding cell cytoplasm of the tissue scetion. Negative result of CD20 not exclude infected with NHL. CD20 and CD30 positive might seen in NHL.

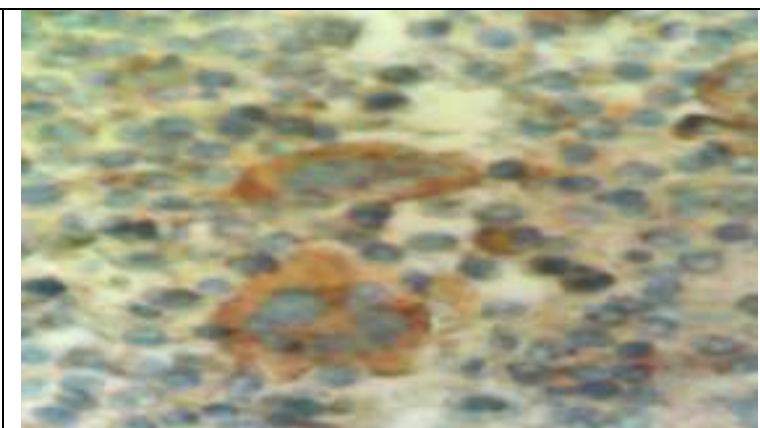

CD 30 positive marker $12: 17(70.58 \%)$ of $\mathrm{HL}$, appear as brownish color surrounding cell cytoplasm of the tissue scetion. Negative result of CD30 not exclude infected with HL . 
Figure ( 3) Positive staining of CD markers ( CD20 , CD30) by using Immunohistochemical staining provided by Dakocytomation company. [8].

\section{Conclusion.}

Present study concluded the fact that cytokines level important to monitored the severity of damage and whether the patients with good or bad prognosis, and refer to important of study other different types of cytokines for Th1, Th2, and NK cell activators, such as ( IL-10, IL-2 and IL-12) to predict of ability to producing pro-inflammatory and anti-inflammatory mediators as well as ability to treatment of such diseases.

Animal models and human studies clearly demonstrate that a physiological role of the immune system is to prevent tumor growth. It is generally assumed that most spontaneous tumors, arising with very long latency times, are indeed poorly immunogenic. Starting with innate immunity, macrophages and other phagocytes are important because they can directly destroy tumor cells and also because they can produce tumor cell fragments that can be picked up by antigen presenting cells. NK cells kill tumor cells with a low MHC expression; they are the sole leukocyte type active in the bloodstream, hence they have an important role in the defense against circulating metastatic cells.

[1] R. Ian, Mackay, and R. Noel, Rose, Autoimmunity and lymphoma: tribulations of B cells . Nature Publishing Grouphttp://immunol.nature.com. 2001.

[2] Irma Airoldi, Robert Gaglidmino, Giuseppe Garr, Anna Cerci one, Franca gerosa, Giuseppe, Guardia. Trinchieri, Vito pistia The interleukin 12 and interleukin 12 receptor system in normal and transformed human B-lymphocytes, hematologica, vol. 87.2002 , $434-442$.

[3] J.M. Wismer, R.C. McKenzie, D.N. Sauder, Interleukin-8 immunoreactivity in epidermis of cutaneous T-cell lymphoma patients. Lymphokine Cytokine Res. Feb;13(1), 1994, 21-7.

[4] M. Watanabe, Y. Ogawa, K. Itoh, "Hypomethylation of CD30 CpG islands with aberrant JunB expression drives CD30 induction in Hodgkin lymphoma and anaplastic large cell lymphoma". Lab. Invest. 88 (1) ,2008, 48-57.

[5] S.J. Park, S. Kim, D.H. Lee, "Primary systemic anaplastic large cell lymphoma in Korean adults: 11 years' experience at Asan Medical Center". Yonsei Med. J. 49 (4), 2008, 601-9.

[6] S. Balamurugan, B. Rajasekar, R.R. Rao, Anaplastic large-cell lymphoma with florid granulomatous reaction: A case report and review of literature. Indian J Pathol Microbiol 52 (1),(2009), 69-70.

[7] Liu, Delong, "Anaplastic Large Cell Lymphoma". Medscape. http: //emedicine. medscape. com/article/208050overview\#aw2aab6c25, 2012.

[8] J.M. Elias, Immunohistopathology : Apractical approach to diagnosis . Chicago; Amer Soc. Clin. Pathol. 1990, Press.46. Cited in Dakocytomation company, manual procedures of Immunohistochemical staining of tissue paraffin sections.

[9] K.W. Moore, A. Ogarra, D.E. Waal -Malefyt, R. P.Vieira, Interleukins Annu. Rev. Immunol, 11, 1993, 165 -190. Cited in Cytokines manual leaflet. S.A. BioSource Europe, Rue De I Nivelles - Belguim, Indousterie, 2011, 8-B-1400 .

[10] F. Frances, B.D. Marchall, () . Overview of Immunodiagnostic Studies : Amanual of Laboratory and Diagnostic Tests . $8^{\text {th }}$ edd. (Lippincott Williams and Wilkins. 2009) 650-663.

[11] L. Gergely, M. Aleksza, L. Váróczy, A. Ponyi , S. Sipka, A. Illés , G. Szegedi, Intracellular IL-4/IFN-gamma producing peripheral T lymphocyte subsets in B cell non-Hodgkin's lymphoma patients. Eur J Haematol. May;72(5) ,2004, 336-41.

[12] H, Zhu, Y. Wang, X. Han, Measurement of five cytokines in the serum of malignant lymphoma patients. Zhonghua Xue Ye Xue Za Zhi. Feb;19(2), 1998,85-8.

[13] D. A Nacinović, S. Stifter , S. Dvornik , Z. Skunca , N. Jonjić , Correlation of serum IL-6, IL-8 and IL-10 levels with clinicopathological features and prognosis in patients with diffuse large B-cell lymphoma. Int J Lab Hematol. Jun;30(3), 2008, $230-9$.

[14] BioGenex , Immunohistochemical atlas solution, version 2.0, San Roman, (2005). 